\title{
Insulin Resistance can be Easily Estimated by Body Mass Index and Waist Circumference in a General Japanese Population
}

\section{To the Editor}

We read with great interest the recent report by Oka and colleagues ${ }^{1)}$, who successfully assessed the association of visceral and subcutaneous adipose tissue with insulin resistance in a Japanese population, using the homeostasis model for the assessment of insulin resistance (HOMA-IR) ${ }^{2)}$ and the Matsuda index ${ }^{3)}$ as indices of insulin resistance/sensitivity. Their detailed investigations also showed that body mass index (BMI) was not inferior in predicting insulin resistance to the area of visceral fat tissue evaluated by computed tomography. Furthermore, interestingly, waist circumference was significantly associated with insulin resistance even after adjustment for visceral fat tissue. These findings indicate the possibility that BMI and waist circumference by themselves would provide considerable information on insulin resistance/sensitivity in a Japanese population. Assessment of insulin resistance/sensitivity is not easy in epidemiological practice. It would be useful if average insulin resistance/ sensitivity of a population could be predictively evaluated using these easy measurements. We therefore investigated the predictive impact of BMI and waist circumference on insulin resistance/sensitivity in a general Japanese population.

We used a database of 1719 Japanese employees of Amagasaki City Office whose fasting plasma glucose levels were $<7.0 \mathrm{mmol} / \mathrm{L}$ and who underwent the $75-\mathrm{g}$ oral glucose tolerance test (OGTT) (UMIN000002391).
None had undergone anti-diabetic treatment and none had malignant neoplasms. They were $50 \pm 9$ years old and 1453 subjects (85\%) were male. Plasma glucose and insulin were measured at 0 and $120 \mathrm{~min}$ during the 75-g OGTT, from which the Matsuda index ${ }^{3)}$ and HOMA-IR ${ }^{2)}$ were calculated. The current analyses were performed after dividing the population randomly into two groups; one group $(n=860)$ was used as the training data, for model derivation, and the other $(n=859)$ as the test data, for model validation.

We first used the training data and developed linear regression equations whose dependent variable was either log-transformed HOMA-IR or the logtransformed Matsuda index (Table 1). As a result, both BMI and waist circumference were significantly associated with log-transformed HOMA-IR $(r=0.45$ and 0.41$)$ and the log-transformed Matsuda index $(r=$ -0.40 and -0.38 ) in univariate analysis (all $p<0.01$ ) (See Model 1 and 2). Furthermore, bivariate analysis showed that BMI and waist circumference were associated with these indices, independently of each other (Model 3). Their significant associations were still observed even after adjustment for age and sex (Model 4 ), as well as other metabolic profiles (Model 5).

We subsequently assessed the model validity of the developed regression equations (Model 1 to 5) using the test data. Estimated average values of HOMA-IR and the Matsuda index were compared to the respective average measurements in every quintile

Table 1. Linear regression model derived from the training data to estimate insulin resistance/sensitivity

\begin{tabular}{|c|c|c|c|c|c|c|c|c|c|c|}
\hline \multirow{2}{*}{$\begin{array}{l}\text { Dependent variable } \\
\text { Explanatory variable }\end{array}$} & \multicolumn{2}{|c|}{ Model 1} & \multicolumn{2}{|c|}{ Model 2} & \multicolumn{2}{|c|}{ Model 3} & \multicolumn{2}{|c|}{ Model 4} & \multicolumn{2}{|c|}{ Model 5} \\
\hline & $r$ & ( $p$ value) & $r$ & ( $p$ value) & $\beta$ & ( $p$ value) & $\beta$ & ( $p$ value) & $\beta$ & ( $p$ value) \\
\hline \multicolumn{11}{|l|}{ Ln HOMA-IR } \\
\hline Body mass index $\left(\mathrm{kg} / \mathrm{m}^{2}\right)$ & 0.45 & $(<0.01)$ & - & & 0.34 & $(<0.01)$ & 0.32 & $(<0.01)$ & 0.21 & $(<0.01)$ \\
\hline Waist circumference $(\mathrm{cm})$ & - & & 0.41 & $(<0.01)$ & 0.13 & $(0.02)$ & 0.15 & $(0.01)$ & 0.14 & $(0.01)$ \\
\hline \multicolumn{11}{|l|}{ Ln Matsuda index } \\
\hline Body mass index $\left(\mathrm{kg} / \mathrm{m}^{2}\right)$ & -0.40 & $(<0.01)$ & - & & -0.29 & $(<0.01)$ & -0.32 & $(<0.01)$ & -0.21 & $(<0.01)$ \\
\hline Waist circumference $(\mathrm{cm})$ & - & & -0.38 & $(<0.01)$ & -0.14 & $(0.01)$ & -0.12 & $(0.04)$ & -0.10 & $(0.05)$ \\
\hline
\end{tabular}

Using training data $(n=860)$, linear regression models were developed whose dependent variables were log-transformed Matsuda index or HOMAIR. Data are correlation coefficients $r$ or standardized partial regression coefficients $\beta$ and their $p$ values. Model 1 , univariate model with body mass index; Model 2, univariate model with waist circumference; Model 3, bivariate model with body mass index and waist circumference; Model 4 , multivariate model with body mass index and waist circumference adjusted for sex and age; Model 5, multivariate model with body mass index and waist circumference adjusted for sex, age, glucose, lipids, and blood pressure. 
A

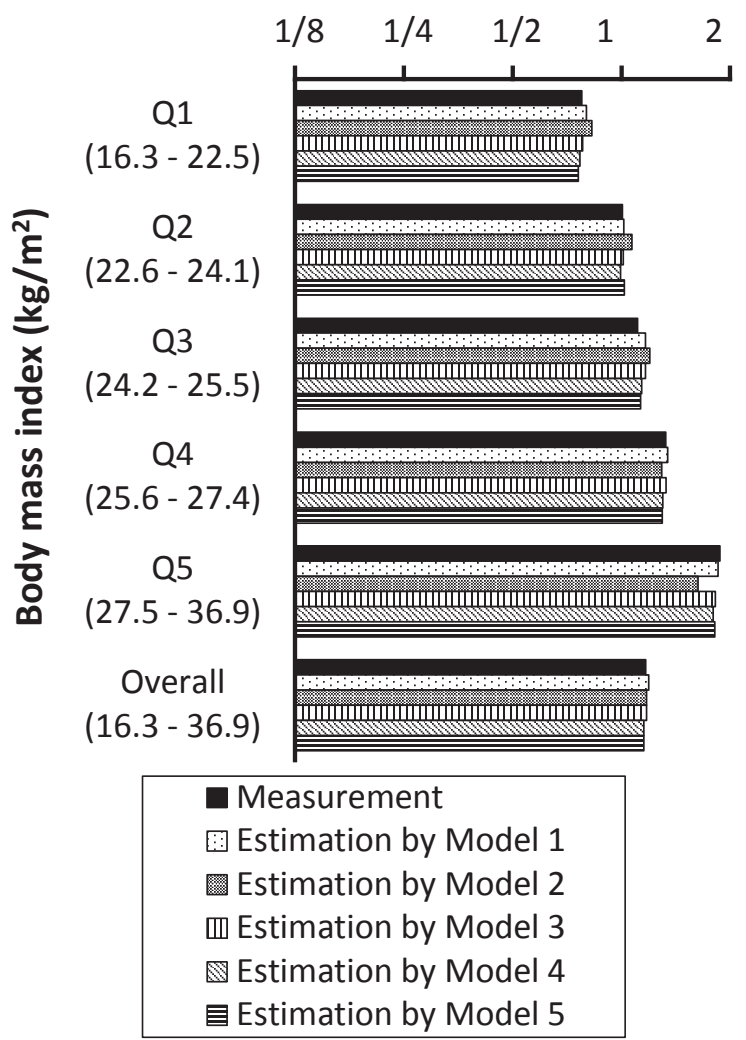

B

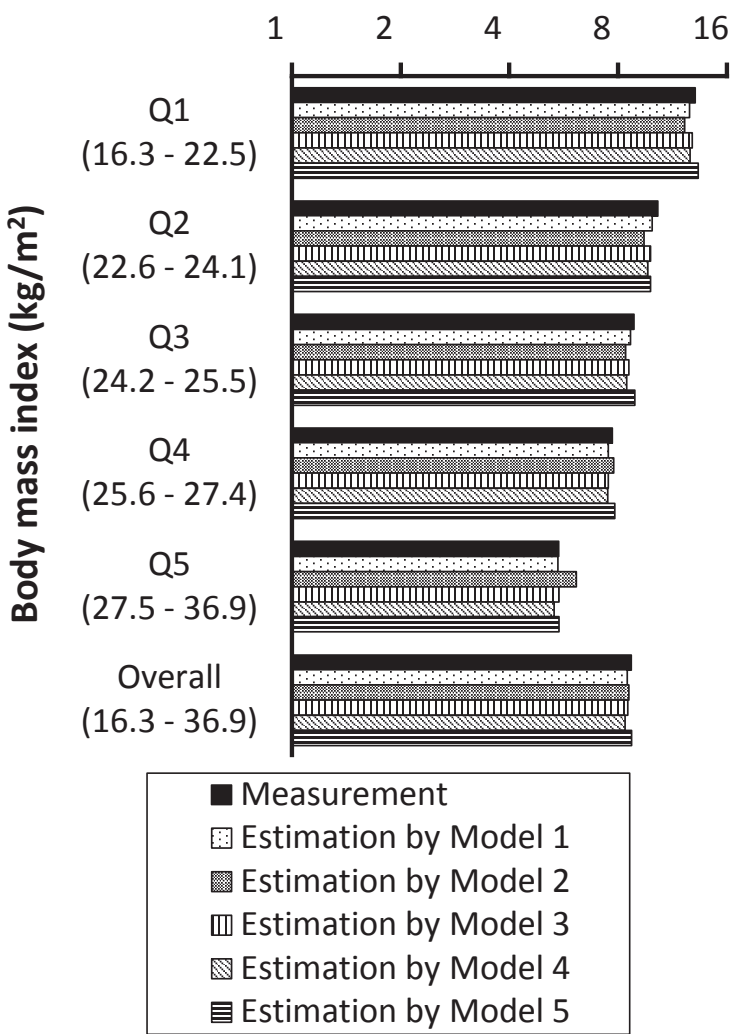

Fig. 1. Measurements and estimations of HOMA-IR (A) and Matsuda index (B) using the test data.

The mean measured and estimated values of HOMA-IR (A) and the Matsuda index (B) are shown for every quintile of body mass index as well as the overall population of the test data $(n=859)$. The estimated values of HOMA-IR and the Matsuda index of each subject in the test data were calculated by assigning his/her values of the explanatory variables to the regression equations developed in the training data (Model 1 to 5 in Table 1). Model 1, univariate model with body mass index; Model 2, univariate model with waist circumference; Model 3, bivariate model with body mass index and waist circumference; Model 4, multivariate model with body mass index and waist circumference adjusted for sex and age; Model 5, multivariate model with body mass index and waist circumference adjusted for sex, age, glucose, lipids, and blood pressure.

of BMI as well as in the overall population of the test data (Fig. 1). As a result, interestingly, all the developed equations, including univariate equations, could estimate the average measurements with similar accuracy (Fig. 1).

Insulin resistance now attracts increasing attention in clinical practice ${ }^{4-6)}$. The current study investigated regression equations for estimating the average measurements of insulin resistance/sensitivity by BMI and/or waist circumference in a general Japanese population. Although insulin resistance/sensitivity is often assessed using HOMA-IR and the Matsuda index in clinical practice, these indices are difficult to measure in epidemiological settings or population-based surveys. Numerous studies have demonstrated that obesity is strongly associated with impaired insulin sensi- tivity or insulin resistance. However, little is known about to what extent some easy and familiar measurements of obesity, i.e., BMI and waist circumference, could provide predictive information for these indices of insulin resistance/sensitivity. We revealed that the average values of HOMA-IR and the Matsuda index could be assessed with considerable accuracy by either BMI or waist circumference.

In a linear regression equation, the dependent variable $y$ can be estimated with a series of explanatory variables $x_{i}$ as follows: $y=\sum_{i} b_{i} x_{i}+a$, where $b_{i}$ and $a$ represent the regression coefficients and intercept. The mean dependent variable of a population $\bar{y}$ can be therefore calculated as: $\bar{y}=\overline{\sum_{\imath} b_{\imath} x_{\imath}+a}=\sum_{i} b_{i} \bar{x}_{i}+a$, indicating that $\bar{y}$ can be easily estimated with a series of average explanatory variables $\bar{x}_{t}$, without an individual dataset of $x_{i}$. 
The current findings suggest that the average HOMAIR and the Matsuda index of a general Japanese population can be predicted using the average BMI or waist circumference.

Previous studies performed detailed measurements of fat distribution and investigated its association with insulin resistance ${ }^{1)}$ and related diseases ${ }^{7-10)}$. However, little is known about whether insulin resistance can be practically estimated with easier measurements. We revealed that HOMA-IR and the Matsuda index were easily estimated by BMI or waist circumference in a general Japanese population. The developed regression equations would be useful for estimation of insulin resistance/sensitivity in epidemiological settings or population-based surveys.

\section{Note}

The developed regression equations for estimating HOMA-IR and the Matsuda index by BMI are as follows:

$\ln ($ HOMA-IR $)=0.095 \times\left[B M I\left(\mathrm{~kg} / \mathrm{m}^{2}\right)\right]-2.210$

$\ln ($ Matsuda index $)=-0.095 \times\left[B M I\left(\mathrm{~kg} / \mathrm{m}^{2}\right)\right]+4.521$

\section{Acknowledgements}

Mitsuyoshi Takahara is a Research Fellow of the Japan Society for the Promotion of Science. The authors thank Midori Noguchi, Amagasaki City Office, Hyogo, Japan, for her great help with data collection.

We declare that we have no conflict of interest.

\section{References}

1) Oka R, Yagi K, Sakurai M, Nakamura K, Nagasawa SY, Miyamoto S, Nohara A, Kawashiri MA, Hayashi K, Takeda Y, Yamagishi M: Impact of visceral adipose tissue and subcutaneous adipose tissue on insulin resistance in middle-aged Japanese. J Atheroscler Thromb, 2012; doi: $10.5551 /$ jat. 12294

2) Matthews DR, Hosker JP, Rudenski AS, Naylor BA, Treacher DF, Turner RC: Homeostasis model assessment: insulin resistance and beta-cell function from fasting plasma glucose and insulin concentrations in man. Diabetologia, 1985; 28: 412-419

3) DeFronzo RA, Matsuda M: Reduced time points to calculate the composite index. Diabetes Care, 2010; 33: e93

4) Gotoh S, Hata J, Ninomiya T, Mukai N, Fukuhara M, Kamouchi M, Kitazono T, Kiyohara Y: Insulin Resistance and the Development of Cardiovascular Disease in a Japanese Community: the Hisayama Study. J Atheroscler

\section{Mitsuyoshi Takahara, Hideaki Kaneto and lichiro Shimomura}

Department of Metabolic Medicine, Osaka University Graduate School of Medicine, Osaka, Japan

Address for correspondence: Hideaki Kaneto, Department of Metabolic Medicine, Osaka University Graduate School of Medicine, 2-2 Yamadaoka, Suita, Osaka 565-0871, Japan

E-mail: kaneto@endmet.med.osaka-u.ac.jp

Received: September 27, 2012

Accepted for publication: November 7, 2012

Thromb, 2012; doi: 10.5551/jat. 13698

5) Wang $\mathrm{H}, \mathrm{Hu}$ B, Feng B: Decreased Beta cell function and insulin sensitivity contributed to coronary artery disease in patients with normal glucose tolerance. J Atheroscler Thromb, 2012; 19: 806-813

6) Uetani E, Tabara Y, Igase M, Kido T, Ochi N, Takita R, Kohara K, Miki T: Liver enzyme and adipocytokine profiles are synergistically associated with insulin resistance: the J-SHIPP study. J Atheroscler Thromb, 2012; 19: $577-$ 584

7) Hiuge-Shimizu A, Kishida K, Funahashi T, Ishizaka $Y$, Oka R, Okada M, Suzuki S, Takaya N, Nakagawa T, Fukui T, Fukuda H, Watanabe N, Yoshizumi T, Ohira T, Nakamura T, Matsuzawa Y, Yamakado M, Shimomura I: Reduction of Visceral Fat Correlates with the Decrease in the Number of Obesity-Related Cardiovascular Risk Factors in Japanese with Abdominal Obesity (VACATION-J Study). J Atheroscler Thromb, 2012; doi: 10.5551/jat.12963

8) Unno M, Furusyo N, Mukae H, Koga T, Eiraku K, Hayashi J: The utility of visceral fat level by bioelectrical impedance analysis in the screening of metabolic syndrome - the results of the Kyushu and Okinawa Population Study (KOPS). J Atheroscler Thromb, 2012; 19: 462-470

9) Nakagawa Y, Kishida K, Funahashi T, Yanagi K, Shimomura I: Coexistence of visceral fat accumulation and sleep-disordered breathing correlates with coronary artery disease. J Atheroscler Thromb, 2012; 19: 728-735

10) Hiuge-Shimizu A, Kishida K, Funahashi T, Okutsu M, Kametani R, Kobayashi H, Nozaki Y, Nomura A, Yokoi H, Yoshizumi T, Ohira T, Nakamura T, Matsuzawa Y, Sumitsuji S, Shimomura I: Coexistence of visceral fat and multiple risk factor accumulations is strongly associated with coronary artery disease in Japanese (the VACATION-J study). J Atheroscler Thromb, 2012; 19: 657663 